\title{
The Adapted Lifestyle-Integrated Functional Exercise Program for Preventing Functional Decline in Young Seniors: Development and Initial Evaluation
}

\author{
Michael Schwenk $^{a, b}$ Ronny Bergquist ${ }^{c}$ Elisabeth Boulton ${ }^{d, i}$ \\ Jeanine M. Van Ancum ${ }^{\mathrm{e}}$ Corinna Nerz ${ }^{\mathrm{a}}$ Michaela Weber ${ }^{\mathrm{b}}$ Carolin Barz $^{\mathrm{a}}$ \\ Nini H. Jonkman ${ }^{\mathrm{e}}$ Kristin Taraldsen ${ }^{c}$ Jorunn L. Helbostad ${ }^{c}$ Beatrix Vereijken ${ }^{c}$ \\ Mirjam Pijnappels ${ }^{\mathrm{e}}$ Andrea B. Maier ${ }^{\mathrm{e}, \mathrm{f}}$ Wei Zhang ${ }^{\mathrm{C}}$ Clemens Becker $^{\mathrm{a}}$ \\ Chris Todd $^{d, h, i}$ Lindy Clemson $^{j}$ Helen Hawley-Hague ${ }^{d, i}$

\begin{abstract}
a Department of Clinical Gerontology and Geriatric Rehabilitation, Robert Bosch Hospital, Stuttgart, Germany; ${ }^{b}$ Network Aging Research, Heidelberg University, Heidelberg, Germany; ${ }^{\circ}$ Department of Neuromedicine and

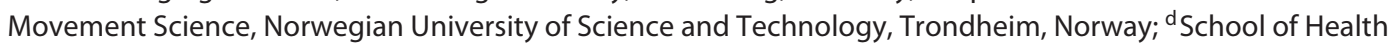
Sciences, University of Manchester, Manchester, UK; ${ }^{\text {} D e p a r t m e n t ~ o f ~ H u m a n ~ M o v e m e n t ~ S c i e n c e s, ~ @ A g e A m s t e r d a m, ~}$ Vrije Universiteit Amsterdam, Amsterdam Movement Sciences, Amsterdam, The Netherlands; ${ }^{\mathrm{f}}$ Department of Medicine and Aged Care, @AgeMelbourne, Royal Melbourne Hospital, University of Melbourne, Melbourne, VIC, Australia; ${ }^{9}$ Laboratory of Movement Analysis and Measurement, École Polytechnique Fédérale de Lausanne, Lausanne, Switzerland; ${ }^{\mathrm{h}}$ Manchester University NHS Foundation Trust, Manchester, UK; 'Manchester Academic Health Science Centre, Manchester, UK; j Faculty of Health Sciences, and Centre of Excellence in Population Ageing Research (CEPAR), The University of Sydney, Sydney, NSW, Australia
\end{abstract}

\section{Keywords}

Healthy aging · Exercise · Prevention · Functional decline · Young seniors

\footnotetext{
Abstract

Background: The Lifestyle-integrated Functional Exercise (LiFE) program is an intervention integrating balance and strength activities into daily life, effective at reducing falls in at-risk people $\geq 70$ years. There is potential for LiFE to be adapted to young seniors in order to prevent age-related functional decline. Objective: We aimed to (1) develop an intervention by adapting Lifestyle-integrated Functional Ex-
}

ercise (aLiFE) to be more challenging and suitable for preventing functional decline in young seniors in their 60 s and (2) perform an initial feasibility evaluation of the program. Pre-post changes in balance, mobility, and physical activity (PA) were also explored. Methods: Based on a conceptual framework, a multidisciplinary expert group developed an initial aLiFE version, including activities for improving strength, neuromotor performances, and PA. Proof-of-concept was evaluated in a 4-week pre-post intervention study measuring (1) feasibility including adherence, frequency of practice, adverse events, acceptability (i.e., perceived helpfulness, adaptability, level of difficulty of single activities), and safety, and (2) changes in balance/mobility (Community

\section{KARGER}

(c) 2019 S. Karger AG, Basel

E-Mail karger@karger.com

www.karger.com/ger
Michael Schwenk, PhD, Research Group Leader

Heidelberg University, Network Aging Research

Bergheimer Strasse 20

DE-69115 Heidelberg (Germany)

E-Mail schwenk@nar.uni-heidelberg.de 
Balance and Mobility Scale) and PA ( 1 week activity monitoring). The program was refined based on the study results. Results: To test the initial aLiFE version, 31 young seniors were enrolled and 30 completed the study (mean age $66.4 \pm$ 2.7 years, $60 \%$ women). Of a maximum possible 16 activities, participants implemented on average $12.1 \pm 1.8$ activities during the intervention, corresponding to mean adherence of $76 \%$. Implemented activities were practiced 3.6-6.1 days/ week and 1.8-7.8 times/day, depending on the activity type. One noninjurious fall occurred during practice, although the participant continued the intervention. The majority found the activities helpful, adaptable to individual lifestyle, appropriately difficult, and safe. CMBS score increased with medium effect size $(d=0.72, p=0.001)$. Increase in daily walking time $(d=0.36)$ and decrease in sedentary time $(d=-0.10)$ were nonsignificant. Refinements included further increasing the task challenge of some strength activities and defining the most preferred activities in the trainer's manual to facilitate uptake of the program. Conclusion: aLiFE has the potential to engage young seniors in regular lifestyle-integrated activities. Effectiveness needs to be evaluated in a randomized controlled trial.

(c) 2019 S. Karger AG, Basel

\section{Introduction}

With a growing population approaching retirement age, there is increasing focus on how to prevent functional decline. Efforts to preserve functional capacity should begin early during the ageing process, when it is possible to maintain skills for independence [1].

While subtle functional decline starts after age 30 , marked performance change occurs after age 60 [2]. This decline is, however, extremely heterogeneous with respect to domains of strength, neuromotor function, and aerobic capacity. Some individuals have relatively preserved function in specific domains, while others have impairments in one or multiple domains $[2,3]$. Low strength is a primary limiting factor for functional independence [3]. Reduced neuromotor functions, including balance, coordination, gait, and agility, frequently cause mobility impairment [3]. Low aerobic fitness and sedentary behavior are all negatively associated with health outcomes $[2,3]$.

Physical activity (PA) is one of the most important determinants for maintaining function during the ageing process [4]. General PA such as walking is associated with better health and function [5]. Reducing sedentary behavior is also important [6]. Further, regular strength and neuromotor training are recommended by the American College of Sports Medicine (ACSM) [4]. However, only $21 \%$ of retired people meet ACSM recommendations to perform strength and neuromotor exercises $\geq 2$ days/ week [7]. Specific interventions fostering regular PA, strength, and neuromotor exercise during ageing are needed.

Current interventions are typically offered as formal programs, with exercises carried out in addition to routine tasks. While effective in the short term, formal programs have largely failed to induce long-term change toward regular exercise [8]. For many seniors, formal exercise is not appealing [9], and everyday activities such as gardening is preferred [10].

Integrating exercises into lifestyle activities has been suggested as a promising alternative or supplement to formal training [11]. Integrated training can be performed with a minimum of additional time and can foster behavioral change by turning daily routines into opportunities to exercise. Some studies have focused solely on general PA such as walking, for example, walking rather than taking the bus. This approach has been expanded to integrate functional strength and balance exercises, as described in the "Lifestyle-integrated Functional Exercise" (LiFE) program [12]. LiFE was developed for falls prevention and comprises age-appropriate lower-extremity strength and balance activities. Compared to traditional training, LiFE has shown higher adherence and greater effects on functional performances in fall-prone adults with an average age of 83 years [12]. LiFE defines a set of increasingly challenging lifestyle-integrated strength activities such as carrying out a half-squat while opening a floor cupboard. Likewise, a set of gradually increasing balance activities is defined. However, these exercises have been developed specifically for older fallers and lack more challenging activities for preventing early functional decline in young seniors. The literature on the development of programs for older adults stresses the importance of adequate challenge and progression [13]. This could include age-appropriate neuromotor activities focusing on agility and sensorimotor function, as defined by the ACSM [7]. Also, challenging strength activities allowing adequate muscle loading for inducing a training effect in young seniors [13], for example, lunging with additional weight, are not included in LiFE. Furthermore, LiFE does not focus on increasing aerobic capacity and reducing sedentary behavior, which have both been shown to be important for healthy ageing $[2,3]$.

LiFE does not contain a catalog of activities with the intention to prevent functional decline in multiple do- 
mains in young seniors and there is therefore likely to be a ceiling effect with the current program. Our systematic review suggests that the program has not been adapted to those aged 60-70 years [11]. Developing an adapted LiFE program seems highly relevant to tackle an accelerated, but preventable, functional decline at this age in those not meeting PA guidelines, not interested in formal training, and those with functional decline risk factors.

The primary aims of this study were (1) to develop a lifestyle-integrated intervention for young seniors by adapting Lifestyle-integrated Functional Exercise (aLiFE) to be more adequately challenging for 60-70-year-olds and (2) to perform initial evaluation of the aLiFE's feasibility. As secondary aims, we explore pre-post intervention changes in balance, mobility, and PA patterns.

\section{Methods}

\section{Development of the aLiFE Program}

aLiFE was developed as part of the PreventIT Project (Horizon 2020, grant no. 689238). PreventIT aims to develop aLiFE (year 1), transfer it to an electronic platform (eLiFE, year 1) and compare both programs in a randomized controlled trial (RCT; year 2-3). This paper reports the development, testing, and refinement of aLiFE. The study protocol relating to eLiFE and the RCT is published separately [14]. We used the UK Medical Research Council (MRC) framework [15] to guide the development and initial feasibility testing of the aLiFE intervention in 2 stages. Stage 1 included the development of the initial aLiFE version based on 5 multidisciplinary workshops involving experts in movement and exercise sciences, physiotherapy, psychology, public health, occupational therapy, gerontology, and geriatric medicine. Six seniors aged between 60 and 70 years ( 2 males, 4 females) were also involved in the initial development and provided feedback about the ideas of the interdisciplinary team. Stage 2 included feasibility testing of the initial aLiFE version in a pilot study with the target group. Refinements to the program were made based on the study results.

\section{Stage 1: Development of the Initial aLiFE Version}

Conceptual Framework

aLiFE targets risk factors for functional decline using personalized lifestyle-integrated training. We do this by integrating risk factor-specific, personalized activities into everyday routines, ultimately becoming habitual. The conceptual framework includes 3 elements: "Target risk factors," "Personalized activities," "Behavioral change."

Target Risk Factors. Literature was used to define risk factors of age-related functional decline that are modifiable via exercise training and PA $[3,16]$. Factors were (i) Function and mobility related lower-extremity strength impairment. (ii) Neuromotor deficits related to postural instability in everyday tasks of young seniors (specifically, deficits in balance, agility, sensorimotor control, and motor-cognitive control). (iii) Functional PA decline and sedentariness that are increasingly prevalent in young seniors.
Personalized Activities. Individual tailoring maximizes challenge and reduces risk. For instance, some young seniors have above average functional strength (sit-to-stand test) comparable to young adults $(6 \mathrm{~s})$ while others' performance is comparable to those aged $\geq 85$ (15 s), putting them at risk of loss of mobility [17]. The intervention needs to offer a range of lower-extremity functional strength activities appropriate to baseline performance, permitting progression in the longer-term. Based on an individualized assessment, strength training should also be tailored to deficits in specific lower-extremity muscle groups relevant for ambulation, including hip, thigh, shank, and foot muscles [3, $4,13]$.

Likewise, some young seniors show largely intact neuromotor abilities, while others have impairments resulting in gait instability and falls [18]. Neuromotor impairments are related to degradations of sensory channels, that is, vestibular, visual, proprioceptive, and deficits in higher level adaptive systems [19]. Reduced dualtask performance also causes postural instability [20]. Accordingly, the program needs to target specific neuromotor risk factors via specific activities tailored based on individualized assessment.

Furthermore, high interindividual differences in PA levels of retired adults require a program offering PAs ranging from basic activities, for reducing sedentary behavior, toward challenging PAs such as fast walking in order to allow progression over the course of the intervention.

In developing the intervention content, we considered established PA and exercise guidelines for (i) progressive strength training of lower extremity muscles relevant for daily activities; (ii) progressive neuromotor training including balance, agility, and coordination to ambulate safely; and (iii) general PA promotion and strategies for reducing sedentary behavior [3, 4, 13, 21].

Behavioral Change. The intervention should be tailored to individuals' needs, preferences, and personal goals; to change behaviors form habits; and promote appropriate activity [22]. We know that goal setting and personalized feedback are motivational for older adults, including those in this age bracket [23]. The habit formation concept has been shown to be feasible and effective in previous interventions such as changing dietary behavior [24] and lifestyle-integrated activity habits [11,22]. Activities preferred by young seniors [25] such as walking, outdoor activities, household tasks, shopping, food/drink preparation, reading, or watching TV were considered for integrating exercises (online suppl. Table 1; see www.karger.com/doi/10.1159/000499962 for all online suppl. material). Individual activity goals were an addition to the original LiFE conceptual framework to foster behavioral change (Table 1). The aLiFE behavior change framework, which builds on the original LiFE framework [22], was underpinned by Habit Formation Theory and the Health Action Process Approach, and comprises 25 behavior change techniques mapped to Michie et al.'s [26] taxonomy, fully described elsewhere [23].

\section{Content of the aLiFE Program}

LiFE comprises basic functional activities for older fallers [12], but needed to be adapted to include both basic and challenging activities for individual tailoring and progression in young seniors. Table 1 gives an overview of characteristics of aLiFE compared to LiFE. All the components of the original program were retained, including the mode of delivering the intervention via home visits by qualified trainers [27]. Activities were extended for aLiFE by adding challenging strength and neuromotor activities. Addition- 
Table 1. Adaptions made in aLiFE as compared to LiFE

\begin{tabular}{|c|c|c|}
\hline Aspect & LiFE & aLiFE \\
\hline Aim of the program & Prevent falls & $\begin{array}{l}\text { Prevent functional } \\
\text { decline }\end{array}$ \\
\hline Target group & $\begin{array}{l}\text { Older adults } 75+\text { years } \\
\text { at risk of falls }\end{array}$ & $\begin{array}{l}\text { Young seniors aged } \\
60-70 \text { years }\end{array}$ \\
\hline \multicolumn{3}{|l|}{ Activity framework } \\
\hline Low-intensity strength tasks & ++ & ++ \\
\hline High-intensity strength tasks & - & ++ \\
\hline \multicolumn{3}{|l|}{ Neuromotor module } \\
\hline Static balance tasks & ++ & ++ \\
\hline Dynamic balance tasks & + & ++ \\
\hline Sensorimotor tasks & + & ++ \\
\hline Dual-tasks & + & ++ \\
\hline Self-perturbation tasks & + & ++ \\
\hline Agility tasks & - & ++ \\
\hline \multicolumn{3}{|l|}{ PA module } \\
\hline \multicolumn{3}{|l|}{ Structured recommendations for reducing sedentary } \\
\hline \multicolumn{3}{|l|}{ Behavioral change framework } \\
\hline Intensions to act & ++ & ++ \\
\hline Goal setting for young seniors & - & ++ \\
\hline Planning how, when, and where & ++ & ++ \\
\hline Activities into daily routine & ++ & ++ \\
\hline Habit formation and mastery & ++ & ++ \\
\hline Situational, environmental cues & ++ & ++ \\
\hline Practice and repetition & ++ & ++ \\
\hline Self-monitoring & ++ & ++ \\
\hline
\end{tabular}

The original LiFE modules were retained in aLiFE but extended by adding challenging activities. A physical activity module was developed for aLiFE. Some adaptions were also made to the behavioral change framework, which are reported in detail elsewhere [23]. - = not included; + = basic features included; ++ advanced features included. aLiFE, adapted Lifestyle-integrated Functional Exercise; LiFE, Lifestyle-integrated Functional Exercise; PA, physical activity.

ally, a module to increase PA and reduce sedentary behavior was developed. The aLiFE activity modules are summarized below.

Strength Activity Module. The aLiFE strength activities focus on lower extremity muscle groups including hip, thigh, and ankle. The 4 LiFE strength principles for increasing frequency ("increase the number of times you use a muscle"), intensity ("use fewer muscles to move the same weight," "increase the amount of weight you have to lift or move"), and duration ("move slowly") were retained. New principles ("increase the range of your motion") and more challenging activities such as one-legged squatting or lunging were added (online suppl. Table 1). In summary, aLiFE comprises 7 strength activities including "bend your knees" (squatting, lunging), "sit-to-stand," "on your toes" (toe walking, toe standing), "on your heels" (heel walking, heel standing), "up the stairs" (stair climbing), "move sideways" (sideways walking), and "tightening muscles" (ankle/hip flexion/extension, wall sitting). Task challenge increases from Levels 1-4, based on one or a combination of strength principles (online suppl. Table 1). For example, squatting progresses from a quarter squat (Level 1), to a half squat (Level 2), to a quarter one-legged squat (Level 3). Levels are individually adjusted based on the aLiFE Assessment Tool (aLAT), administered by a trainer based on standardized procedure (online suppl. Table 2 ). The aLAT is used to set starting levels as well as upgrading activities.

Neuromotor Activity Module. The original LiFE program comprises balance activities categorized into the principles (1) "reducing base of support," (2) "shifting weight and moving to limits of stability," and (3) "stepping over objects." Task challenge in original LiFE ranges from practicing activities with hold support to practicing with one additional task which is either cognitive (e.g., counting backward) or manual (e.g., combing hair; online suppl. Table 1). 
The original LiFE balance training was retained in aLiFE and extended by adding neuromotor training comprising balance, agility, sensorimotor, and multitask training relevant for young seniors $[4,6]$. The aLiFE neuromotor activity module includes 7 activities categorized into 4 principles: (1) "reducing base of support" (3 activities), (2) "shifting weight and moving to limits of stability" (one activity), (3) "stepping over objects" (one activity), and (4) "stepping and hopping in different ways" (2 activities; online suppl. Table 1). For principles 1-3, task challenge ranges from practicing activities with hold support (level 1) to practicing activities with 2 additional challenges (level 4). Additional challenges include manual (e.g., brushing hair), sensorimotor (e.g., eyes closed), cognitive (e.g., talking on phone), or self-perturbation (e.g., pivot turn) tasks. Additional challenges can be combined in several ways such as one-leg stand with eyes closed plus cognitive task. Progression is based on motor-learning theory [28] starting with focusing consciously on the primary activity such as one-leg stand with support (level 1). If the task can be executed safely, additional challenges are added (level 3-4). Ecologically valid challenges foster practice of relevant functional abilities. For instance, turning the head and performing a manual activity while maintaining balance are typical everyday tasks requiring simultaneous coordination of motor skills.

The principle "stepping, hopping, and jumping" comprises agility activities specifically developed for aLiFE (online suppl. Table 1). These activities are based on square-stepping exercises [29], which are age-relevant challenges and can be incorporated into everyday routine (e.g., during walking). For aLiFE, a series of stepping patterns with increasing task challenge were developed (online suppl. Table 1; Fig. 1,2). Task challenges can be further increased by progressing from stepping to hopping, to jumping. Stepping patterns are taught by a trainer during home visits and afterward implemented into everyday routines. Stepping activities provide agility exercises during everyday activity, with simultaneous neuromuscular, cardiocirculatory, and cognitive training [29]. Training levels for all neuromotor activities are personalized using aLAT (online suppl. Table 2).

PA Module. A stepwise increase of PA and reduction in sedentary time conceptual model were developed for aLiFE, taking into account that our younger target population are more active outdoors than the original LiFE population [30]. Following the LiFE model, PA can be integrated into daily life without requiring much additional time. Principles are based on contemporary evidence about health benefits of increasing PA and reducing sedentary behavior $[6,21]$. The aLiFE PA-module comprises 2 principles: "Walk more" and "Reduce sedentariness." Two types of activities are described within each principle: "Walk more" covers walk longer and walk faster and "Reduce sedentariness" comprises sit less and break up sitting (online suppl. Table 1). The participant can plan specific situations or cues for when to perform these activities (e.g., get up during TV commercial break, or park the car further from the grocery store to walk further). When a participant chooses an activity from the PA module, and is able to improve walking distance/pace and/or sitting time/breaks, he or she is encouraged to progress by increasing levels, or by finding additional situations into which the activities can be integrated.

The original LiFE participants' [31] and trainers' manuals [27] were adapted by including background information, explanations, and pictures of aLiFE principles and activities.
Stage 2: aLiFE Feasibility Pilot

Study Design and Setting

A multicenter pilot study was run at the Robert-Bosch-Hospital Stuttgart (Germany), VU University Medical Centre Amsterdam (Netherlands), and the Norwegian University of Science and Technology (Norway). It comprised a 4-week, uncontrolled, prepost design to test the feasibility of aLiFE and explore the potential for changes in balance, mobility, and PA. The intervention lasted 4 weeks including 4 home visits. This was shorter than the original LiFE (7 visits over 3 months) [12], but sufficient for initial intervention testing. The study was conducted in 2016; recruitment (March - April), baseline assessment (May - June), intervention (May - July), and follow-up assessment (June - July). Ethical approvals and registration (ISRCTN37750605) were obtained.

\section{Study Population}

Each site aimed to recruit 10 participants via newspapers and flyers, total $=30$. We aimed to recruit participants aged 60-70 living in the community who were not frequently exercising. Exclusion criteria were inability to walk $500 \mathrm{~m}$ without aids, cognitive impairment (Montreal Cognitive Assessment $<24$ points [32]), diseases where exercise is contraindicated, and attending organized exercise classes more than twice a week and/or exercising $>2 \mathrm{~h}$ alone each week. Participants provided written informed consent.

\section{Study Procedures}

Assessments. Pre/post-assessments were conducted at study centers, except daily PA assessment (described below).

Administering the Intervention. aLiFE was administered following original LiFE guidelines [27], except for the shorter intervention duration and fewer home visits. One week prior to the first visit, participants received the original LiFE Daily Routine Chart (DRC) to document weekly personal routines (e.g., housework, shopping, leisure activities). DRC information was used during visits to identify everyday tasks for integrating activities [27]. Participants were asked to read the background section of the aLiFE participants' manual, provided before the first visit. Each visit lasted approximately $1.5 \mathrm{~h}$. Visits were conducted by aLiFE trainers certified through a 2-day course (online suppl. Table 3 ). Six trainers (2 each in Stuttgart, Amsterdam, Trondheim) with a background in physical therapy or exercise science taught the program. The first visit comprised reviewing the DRC and performing the aLAT to set the difficulty level for activities (online suppl. Table 2). Trainer and participant agreed on up to 4 activities, depending on each participant's preferences. These were documented in an Activity Planner, including "how" (e.g., without holding support), "when" (e.g., while brushing teeth), and "where" (e.g., in the bathroom) to perform the activities. Trainers recommended selecting activities from different modules including strength, neuromotor, and PA. Following the LiFE model, trainers recommended practicing the prescribed activities as often as possible each day, but no number of repetitions was specified. Subsequent visits included implementation of up to 4 new activities, depending on participants' preferences. Participants were encouraged to try as many activities as possible in order to experience the program.

Measurements

Participant Characteristics. Comorbidities (number), fall history in the past 6 months, cognition (Montreal Cognitive Assess- 
Table 2. Baseline characteristics of study participants $(n=31)$

\begin{tabular}{lc}
\hline Variable & $n=31$ \\
\hline Age, years & $66.4 \pm 2.7(60-70)$ \\
Women, number & $20(60)$ \\
MoCA (0-31), score & $26.7 \pm 2.0(24-30)$ \\
Comorbidities, number & $1.7 \pm 1.2(0-4)$ \\
Falls in the past 6 months, number of participants & $7(22.6)$ \\
Pain during walking, Visual Analogue Pain Scale (0-10), score & $2.2 \pm 1.9(0-7)$ \\
30-s chair stand, number of repetitions & $12.8 \pm 2.6(9-19)$ \\
TUG, s & $8.5 \pm 1.6(5.4-11.7)$ \\
CBMS (0-96), score & $66.4 \pm 12.7(28-86)$ \\
Activities-specific Balance Confidence Scale (0-100), score & $88.5 \pm 10.8(59-99)$ \\
400 m walk, s & $329 \pm 50(227-424)$
\end{tabular}

Data are presented as mean \pm SD (range) or $n(\%)$. MoCA, Montreal Cognitive Assessment; TUG, TimedUp-and-Go; CBMS, Community Balance and Mobility Scale.

ment), pain during walking (Visual Analogue Scale), and balance confidence (Activities-specific Balance Confidence Scale [33]) were assessed. Functional lower extremity strength was measured by the number of repetitions achieved during 30-s chair stand [34]. Functional performance was measured using Timed-Up-and-Go test [35]). The Community Balance and Mobility Scale (CBMS) was used to measure balance and mobility in 13 challenging tasks representing functional skills required for safe ambulation in relatively fit seniors [36]. Aerobic capacity was measured using the $400 \mathrm{~m}$ walk at a fast pace (20 times in a $20 \mathrm{~m}$ loop).

Feasibility measures obtained during intervention:

- Adherence defined as the number of activities implemented during the intervention, with a maximum of 16 (4 per visit). "Implemented" was defined as reporting performing an activity at least once per week.

- Frequency of practice defined as weekly practice of each activity reported in the Activity Planner. Daily practice was documented as activity episodes in an Activity Counting Sheet [27]. An activity episode represents an aLiFE activity integrated into a daily task. For instance, tandem walking along the hallway would be one episode.

- Adverse events including self-reported pain, falls and injuries were collected by the trainers.

- Acceptability of the activities defined as reported perceived helpfulness for improving strength, balance, and PA; adaptability to personnel routine, and safety of practice documented by 7-point Likert-scale items developed for the study administered during each visit.

- Task challenge of aLiFE practice was documented in the aLAT during visit 1 (online suppl. Table 2). For each activity, the challenge level was documented. Being unable to perform an activity at the lowest level indicated a floor effect, conversely performance at the highest level indicated ceiling effect. Feasibility measures obtained post intervention:

- Acceptability of aLiFE defined as overall reported acceptability, perceived helpfulness, adaptability, level of difficulty, and safety documented using 7-point Likert-scale (online suppl. Table 4). Participants were also asked the open-ended question
"Please explain why you scored in this way and suggest any changes to the program" and answers documented. Focus groups were conducted to collect further information about the aLiFE intervention with results reported elsewhere [37].

- Activity preferences were documented by the question: "Please name your 3 favorite aLiFE activities."

Exploratory pre-post measures:

- Challenging balance and mobility performances measured by the CBMS.

- PA captured in the week prior (week 0 ) and during (week 3 ) the intervention using a sensor worn continuously on the lower back using an elasticated belt (DynaPort MoveMonitor, McRoberts, Netherlands). Participants were instructed only to remove the sensor during water emersion. Percentage of sedentary time (i.e., energy expenditure $\leq 1.5 \mathrm{MET}$ ) and walking time were extracted from raw data using validated algorithms [38]. Assessments were conducted by trained staff, who also delivered the intervention.

\section{Statistical Analysis}

Participant characteristics are reported using parametric or nonparametric measures of central tendency and dispersion (mean SD, median interquartile range) as appropriate. Pre-post changes were explored using Wilcoxon signed-rank test. Mean difference, CIs, and Cohen's d effect sizes were calculated [39]. Effects were interpreted as small $(\mathrm{d}=0.2)$, medium $(\mathrm{d}=0.5)$, and large $(\mathrm{d}=0.8)$, $p$ value $\leq 0.05$ was accepted. Analysis was performed using SPSS 22.0 (IBM, Armonk, NY, USA).

\section{Results}

Sixty-one community dwellers were screened for eligibility (online suppl. Fig. 3), and 31 (10 each in Amsterdam and Trondheim, 11 in Stuttgart) were recruited (Table 2). Timed-Up-and-Go mean $=8.5 \pm 1.6 \mathrm{~s}$, with nobody above 
Table 3. Activities implemented during the intervention

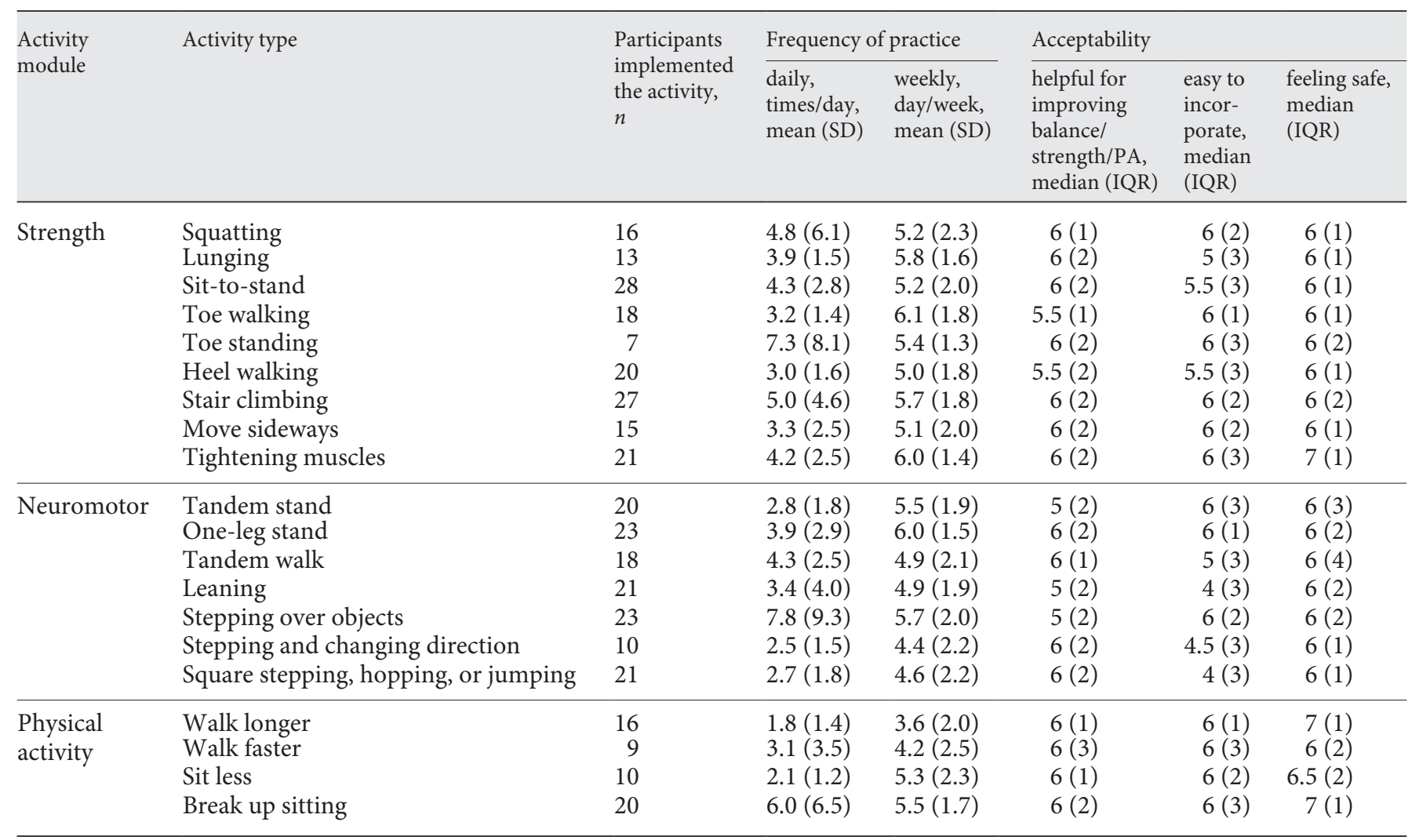

Given are the activities implemented during the intervention period, including frequency of implementation, frequency of practice, and rating of acceptability (helpfulness, adaptability, safety). IQR, interquartile range; PA, physical activity.

the impairment threshold of $12 \mathrm{~s}$ [35]. Their 30-s chairstand mean $=12.8 \pm 2.6$ repetitions, with 12 participants below normal threshold [34]. One person dropped out after baseline assessment, reportedly because the intervention content did not match her primary goal; improving upper extremity strength. All others completed assessments and training. Due to technical errors leading to insufficient sensor data, some PA measurements were lost, and 8 participants were excluded from analysis (week $0 n=5$, week $3 n=3$ ).

\section{Adherence and Frequency of Practice}

Of a maximum possible 16 activities, participants implemented mean $=12.1 \pm 1.8$ activities (range $8-15$ ) during the intervention, corresponding to mean adherence of 76\% (range 50-94\%). Strength activities were most frequently implemented (mean $=5.6 \pm 1.2$, range $3-7)$, followed by neuromotor activities (mean $=4.6 \pm 1.1$, range $2-7)$ and PA (mean $=1.9 \pm 0.6$, range $1-4)$.
Most frequently implemented was the sit-to-stand for the strength module, one-leg stand and stepping over objects for the neuromotor module, and break up sitting for the PA module; while least frequently implemented were toe standing, stepping and changing direction, and walk faster (Table 3).

Weekly frequency of practice ranged between 3.6 and 6.1 days/week depending on the activity (Table 3 ). Highest frequencies were reported for toe walking (strength module), one-leg stand (neuromotor module), and break up sitting (PA module). Lowest frequencies were reported for heel walking, stepping and changing direction, and walk longer.

Daily frequency of practice ranged between 1.8 and 7.8 times per day depending on the activity (Table 3). Highest frequency was reported for toe standing (strength module), stepping over objects (neuromotor module), and break up sitting (PA module). Lowest frequency was reported for heel walking, stepping and changing direction, and walking longer. 


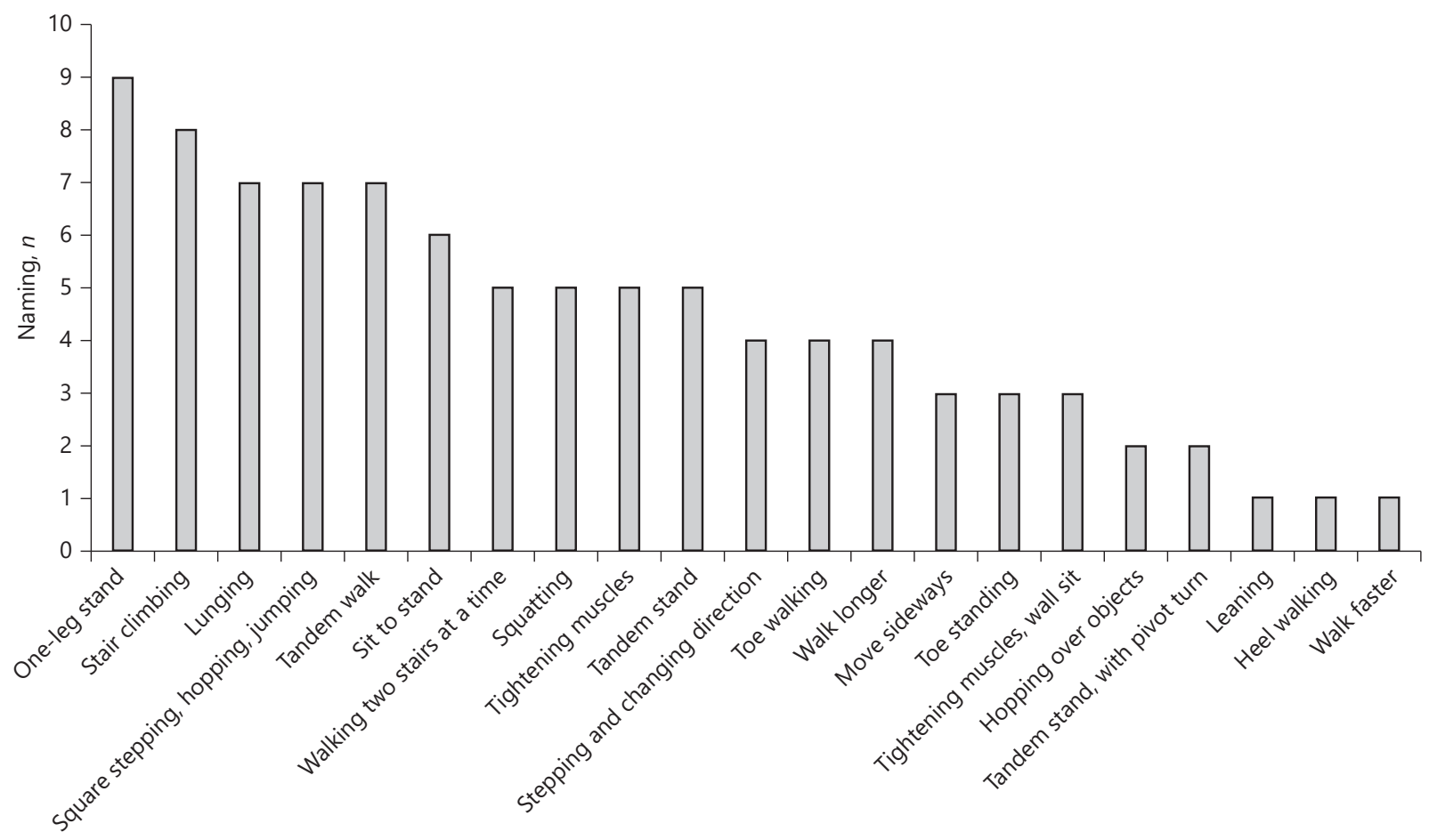

Fig. 1. Participants' rank reported preferences for different activities. Diagrammed are participants' 3 favorite aLiFE activities.

\section{Adverse Events}

Twelve participants reported some pain related to muscle soreness early after initiating the intervention. None reported worsening pain or prolonged exercise-related symptoms. One participant reported a noninjurious fall while performing a stepping activity, reportedly because a tile was loose on the floor, but continued the intervention.

\section{Acceptability of the Activities}

Strength, neuromotor, and PA activities were perceived as helpful for achieving their intended outcomes (Table 3), with one-leg stand, tandem walk, and stepping reportedly perceived as the most helpful neuromotor activities.

Strength activities were reported as easy to incorporate. For neuromotor activities, tandem stand, one-leg stand, and stepping over objects were reported as easy to incorporate. Leaning and stepping, hopping, and jumping were reported as more difficult to integrate. PAs were reported as easy to incorporate. All activities were perceived as safe.

\section{Acceptability of the aLiFE Program}

All except one participant indicated they would recommend aLiFE to a friend. There was strong consensus and most participants liked aLiFE (online suppl. Table 4). Less consensus was found for the adaptability of the activities; some found it challenging while others found it rather easy.

There were positive statements to the open-ended question about aLiFE related to the integrative training approach, including the activities, anticipated benefits, and the trainer (online suppl. Table 5). Suggestions for improvement related to personalization of activities which some participants perceived as too easy, while for others they were too challenging. A number mentioned that too many activities were implemented during a short intervention period. Other aspects that should be improved were lack of social interaction, extensive paperwork, and the short intervention duration.

\section{Activity Preferences}

The one-leg stand was most often mentioned as the favorite activity, followed by stair climbing, lunging, step- 
Table 4. Pre-post differences in balance and mobility and PA

\begin{tabular}{|c|c|c|c|c|c|}
\hline Item & Pre score & Post score & $\begin{array}{l}\text { Mean difference } \\
(95 \% \mathrm{CI})\end{array}$ & Effect size $^{\mathrm{a}}$ & $p$ value $^{\mathrm{b}}$ \\
\hline CBMS, total score $(n=30)$ & $65.97 \pm 12.73$ & $70.41 \pm 13.06$ & $4.44 \pm 6.19(2.10$ to 6.89$)$ & 0.72 & 0.001 \\
\hline Walking time, $\%$ of total daily activity $(n=22)$ & $8.99 \pm 2.01$ & $9.69 \pm 3.04$ & $0.70 \pm 1.92(-0.82$ to 2.22$)$ & 0.36 & 0.131 \\
\hline Sedentary time, $\%$ of total daily activity $(n=22)$ & $44.96 \pm 5.84$ & $44.47 \pm 5.46$ & $-0.48 \pm 4.70(-3.82$ to 2.86$)$ & -0.10 & 0.355 \\
\hline
\end{tabular}

Results of the CBMS and the sensor-based PA assessment (Dynaport) including walking and sedentary time. PA was assessed before (pre) and in week 3 of the intervention (post). Data are mean \pm SD. PA, physical activity; CBMS, Community Balance and Mobility Scale.

${ }^{a}$ Effect size calculated as mean difference/SD of mean difference.

${ }^{\mathrm{b}} p$ value for Wilcoxon-signed rank test.

ping, hopping, and jumping (Fig. 1). Sit less, break up sitting, and heel standing were not favorite activities.

\section{Task Challenge of aLiFE Practice}

aLAT testing revealed that neuromotor activities could be performed by $45-100 \%$ participants at level 1, 45-93\% at level $2,3-77 \%$ at level 3 , and $0-45 \%$ at level 4 , depending on the activity (online suppl. Table 2). Strength activities could be performed by $92-100 \%$ at level $1,74-$ $100 \%$ at level 2, 70-96\% at level 3, and $48-96 \%$ at level 4 , depending on the activity.

\section{Exploratory Pre-Post Measures of Function and PA}

CBMS scores increased from pre-to-post with medium effect size $(\mathrm{d}=0.72, p=0.001)$. Increase in walking time $(\mathrm{d}=0.36)$ and decrease in sedentary time $(\mathrm{d}=-0.10)$ were nonsignificant (Table 4).

\section{Discussion}

Overall, aLiFE was well accepted in our sample. Participants reportedly practiced several activities in the domains of strength, neuromotor exercise, and general PA, suggesting feasibility of the initial aLiFE version. Measureable effects in balance and mobility suggest the potential for improved functional capacity, but a RCT is needed before any strong conclusions can be drawn.

\section{Adherence and Frequency of Practice}

In line with original LiFE, aLiFE trainers offered up to 4 activities per visit and participants decided how many to implement. On average, $76 \%$ of the maximum possible activities were implemented. Based on participants' feedback, the short intervention period made it challenging to implement more activities. Greater ad- herence might be achieved by delivering aLiFE over a longer period.

Higher exercise frequency is generally associated with greater effect [40]. In line with original LiFE, our trainers recommended practicing activities as often as possible, without specifying number of repetitions. Observed weekly frequency (Table 3 ) is comparable to previous research with older cohorts reporting 4.9 days/week of LiFE practice [41]. Results show that a similar level of adherence can be achieved in aLiFE, suggesting that transfer to young seniors was successful.

The lower weekly frequency found for more challenging activities in the neuromotor (i.e., stepping, hopping, jumping) and PA (i.e., walk longer, walk faster) domains might be related to the specific situations where these activities are implemented. As these activities are typically performed outdoors, daily practice may not have been possible. In contrast, activities such as a one-leg stand can be integrated into indoor and outdoor tasks, which may explain their higher frequency.

\section{Adverse Events}

Exercise has numerous positive effects. However, muscle soreness was frequently reported by our participants, most likely because they had not exercised frequently prior to the intervention [42].

Challenging exercises, although generally more effective, are associated with higher incidence of adverse events [43]. Therefore, safety considerations are relevant. The single reported fall event was blamed on a "loose tile" rather than the program but, nonetheless, it is essential to minimize fall risk as a consequence of taking part in aLiFE. The manuals were updated accordingly. 


\section{Acceptability of Activities}

Overall, activities were well accepted, but differed depending on activity type. Activities with self-explanatory effect, such as one-leg stand, were reported as more helpful compared to those focusing on specific aspects of balance control such as leaning, where the outcome was less obvious. Leaning needs to be challenging and toward the limits of stability. Precise execution using ankle joint movement is crucial to produce an effect. Our results suggest that it was difficult to integrate such specific activities. Toe and heel walking may not have been adequately challenging, as, respectively, 96 and $93 \%$ of the participants were able to perform level 4 , suggesting upgrading is needed.

Most activities were rated as easy to incorporate, except stepping activities. Stepping activities require specific environments, such as hallway with adequate space, or a park, and specific situations, such as going for a walk, which may not be part of everyone's daily routine. Despite these challenges, stepping was frequently mentioned as a preferred activity, suggesting it is meaningful for young seniors.

\section{Acceptability of the aLiFE Program}

Overall, high acceptability was reported. Positive ratings were either related to specific aLiFE features or generic exercise effects such as health benefits. Specific attention should be paid to individual tailoring, as some found activities too challenging, while others found them too easy. Tailoring would be better achieved within the 3 months intervention period of the original LiFE program.

Implementing and upgrading activities over the course of the intervention was reported as challenging by some participants. As this process requires time and resources, the short intervention period of this pilot feasibility study may have prevented optimal implementation. A longer period may allow the establishment of an individual program and the habits of integrating activities into daily routines. In our study, summer holidays and traveling were identified as challenges for implementation because they disrupted routine. This suggests that seasons need to be considered as part of the planning process.

Paperwork related to LiFE has been previously discussed as challenging [11], although it is important for behavioral change. Our study participants were asked to ensure they recorded activities accurately, and adherence documentation was collected by trainers during each home visit; this was seen as an additional burden by some participants.

The aLiFE Program

\section{Activity Preferences}

As well as the one-leg stand, challenging activities developed specifically for aLiFE such as stepping, hopping, jumping, and lunging where favorably rated, suggesting they are attractive for young seniors. In contrast, activities for reducing sedentary behavior were not favorably rated, even if practiced frequently. Participants may have performed these activities for expected health benefits [44], but did not seem to find these activities as appealing as more physically challenging strength and neuromotor activities. These perhaps provide more immediate physiological feedback consistent with enhancing self-efficacy beliefs.

\section{Task Challenge of aLiFE Practice}

In our group of relatively fit young seniors, we were able to test whether the activities were challenging enough. Task challenge of the neuromotor activities was well balanced across the 4 levels, suggesting that individually tailoring and progressing within this heterogeneous population is possible. Some strength activities showed ceiling effects, and further upgrading is needed.

\section{Exploratory Pre-Post Measures of Function and PA}

The effect $(\mathrm{d}=0.72)$ observed in CBMS performance may suggest a clinically relevant effect on balance and mobility performance relevant for young seniors, although this needs verification in an RCT. This finding is in line with comparable effect sizes $(\mathrm{d}=0.6)$ for shortterm interventions (3-4 weeks) on balance performance [45].

A systematic review of short-term PA interventions in middle-aged to older adults reports a moderate effect size $(d=0.28)[46]$. A similar, nonsignificant, effect $(d=0.36)$ was observed in this study for daily walking duration. It is not clear whether nonsignificance here is related to limited effect or small sample size, and a fully powered RCT is needed. At baseline, participants spent $9 \%$ of total daily activity time walking, indicating they were quite active [47]. Increasing PA further via aLiFE may have been unrealistic. Our short intervention may have had limited success in implementing PA into routines as this requires reorganization, for example, walking instead of taking the bus, and is likely better achieved over a longer period.

Conflicting results have been reported for interventions reducing sedentary behavior in older adults [48]. We found a nonsignificant $1 \%$ reduction for sedentary time. While no conclusion about effectiveness can be drawn from this feasibility study, our results may indicate greater challenges in influencing sedentary behavior 
compared to other PA domains, such as strength or neuromotor activities. On the same note, participants reported frequent implementation of the break up sitting activity designed for reducing sedentary behavior. Practice may have resulted in multiple, short nonsedentary bouts which, however, did not lead to measurable reduction of total sedentary time. Nevertheless, breaking up prolonged bouts of sitting is beneficial for reducing cardiovascular risk [49]. An RCT is required to further explore changes in function and PA, with an objective assessment of single sedentary episodes to evaluate the impact of aLiFE.

\section{Refinement of aLiFE}

Based on the present results, strength activities have been further upgraded by introducing half one-legged squat at level 4, doubling distance of toe and heel walking, and introducing a new level 5 whereby participants can further adjust task challenge using strategies such as increasing isometric hold time, for example, for squatting, and increasing distance for toe and heel walking. Preferred activities were specified in the trainer manual for prioritization at the start of the intervention. A section about muscle soreness and pain has been included in the manuals, to interpret whether symptoms are indicative of an initial training effect, or require medical attention. Advice on avoiding falls during practice, including safe environments, floor conditions, and footwear, has also been included.

\section{Limitations}

The short intervention period was sufficient for initial feasibility testing and identification of factors for further iterative development according to the MRC framework, but not for evaluating effectiveness, behavior change or full adoption. Implementing a relatively high number of activities within a short period may have overtaxed some participants, limited appropriate upgrading over time, and negatively biased reporting. No information about upgrading was collected, to limit participant burden. We did not measure whether the principles "walk longer" and "walk faster" resulted in sufficiently challenging activities with adequate duration and intensity for inducing an effect on health. The uncontrolled design prevents drawing any robust conclusion about effectiveness. Our findings in relatively fit young seniors may not be generalizable to those with more advanced functional decline. However, we retained all original LiFE activities suggesting that aLiFE is also feasible in more impaired persons, through its tailored approach.

\section{Conclusion}

The first 2 steps of adapting LiFE to young seniors were successfully completed and the positive overall feedback justifies continuation. Study results allow program refinement and the measureable effects are encouraging, but require RCT-based validation. According to the MRC framework, the next step will be to test implementation of aLiFE in a larger, longer duration feasibility RCT [14]. Furthermore, the effectiveness and costs of aLiFE need to be compared to formal programs. We will evaluate strategies for a potentially more cost-effective ICT-based intervention delivery of aLiFE [14] and a group model for teaching the program [50]. In summary, the present study represents an important step toward the development of an integrated training approach for preventing functional decline in young seniors. It should be seen as an important part of the process of intervention development.

\section{Acknowledgments}

We thank the developers of the original LiFE program from the University of Sydney, Maria Fiatarone Singh and Jo Munro, along with co-author L.C., for allowing us to develop aLiFE. We thank Siri Lund Gjerde, Synnøve Ulseth, and Isis Huisman for their help in performing the aLiFE study.

\section{Statement of Ethics}

Participants have given written informed consent. The study protocol has been approved by the relevant research institutes' committees on human research: Stuttgart, Ethik-Kommission am Universitätsklinikum Tübingen, 033/2016BO2; Amsterdam, Medical Ethical Committee, VU University Medical Center, NL56456.029.16; Trondheim, Regional Committees for Medical and Health Research Ethics of the Norwegian University of Science and Technology, REC Central 2016/48.

\section{Disclosure Statement}

L.C. owns the copyright of the original LiFE program. Other authors have no conflict of interest to declare.

\section{Funding Sources}

This work was supported by the European Commission's Horizon-2020 research and innovation program (PreventIT Project, grant number 689238). The content of this paper is solely the responsibility of the authors and does not necessarily represent the official views of the European Commission. 


\section{Author Contributions}

M.S.: development of concept and design, study management, statistical analysis, interpretation of data, and preparation of manuscript. R.B., K.T., C.N., J.V.A., and N.J.: development of concept and design, study management, and data acquisition. E.B., H.H.H., K.T., B.V., J.L.H., A.B.M., M.P., C.B., C.T., and L.C.: development of concept and design. All authors contributed to interpretation of data, drafting the article, and final approval of the version to be published.

\section{References}

1 Landi F, Calvani R, Tosato M, Martone AM, Fusco D, Sisto A, et al. Age-related variations of muscle mass, strength, and physical performance in community-dwellers: results from the milan expo survey. J Am Med Dir Assoc. 2017 Jan; 18(1):88.e17-24.

2 Ferrucci L, Cooper R, Shardell M, Simonsick EM, Schrack JA, Kuh D. Age-related change in mobility: perspectives from life course epidemiology and geroscience. J Gerontol A Biol Sci Med Sci. 2016 Sep;71(9):1184-94.

3 Bauman A, Merom D, Bull FC, Buchner DM, Fiatarone Singh MA. Updating the evidence for physical activity: summative reviews of the epidemiological evidence, prevalence, and interventions to promote "active aging". Gerontologist. 2016 Apr;56 Suppl 2:S268-80.

4 Garber CE, Blissmer B, Deschenes MR, Franklin BA, Lamonte MJ, Lee IM, et al.; American College of Sports Medicine. American College of Sports Medicine position stand. Quantity and quality of exercise for developing and maintaining cardiorespiratory, musculoskeletal, and neuromotor fitness in apparently healthy adults: guidance for prescribing exercise. Med Sci Sports Exerc. 2011 Jul;43(7):1334-59.

5 McPhee JS, French DP, Jackson D, Nazroo J, Pendleton N, Degens H. Physical activity in older age: perspectives for healthy ageing and frailty. Biogerontology. 2016 Jun;17(3):567-80.

6 Ekelund U, Steene-Johannessen J, Brown WJ, Fagerland MW, Owen N, Powell KE, et al.; Lancet Physical Activity Series 2 Executive Committe; Lancet Sedentary Behaviour Working Group. Does physical activity attenuate, or even eliminate, the detrimental association of sitting time with mortality? A harmonised meta-analysis of data from more than 1 million men and women. Lancet. 2016 Sep;388(10051):1302-10.

7 Merom D, Pye V, Macniven R, van der Ploeg $\mathrm{H}$, Milat A, Sherrington C, et al. Prevalence and correlates of participation in fall prevention exercise/physical activity by older adults. Prev Med. 2012 Dec;55(6):613-7.

8 Hill KD, Hunter SW, Batchelor FA, Cavalheri $\mathrm{V}$, Burton E. Individualized home-based exercise programs for older people to reduce falls and improve physical performance: A systematic review and meta-analysis. Maturitas. 2015 Sep;82(1):72-84.

9 Costello E, Kafchinski M, Vrazel J, Sullivan P. Motivators, barriers, and beliefs regarding physical activity in an older adult population. J Geriatr Phys Ther. 2011 Jul-Sep;34(3): 138-47.
10 Burton E, Lewin G, Boldy D. Physical activity levels of older adults receiving a home care service. J Aging Phys Act. 2013 Apr;21(2):140-54.

11 Weber M, Belala N, Clemson L, Boulton E, Hawley H, Becker C, et al. Feasibility and effectiveness of intervention programmes integrating functional exercise into daily life of older adults: A systematic review. Gerontology. 2018;64(2):172-87.

12 Clemson L, Fiatarone Singh MA, Bundy A, Cumming RG, Manollaras K, O’Loughlin P, et al. Integration of balance and strength training into daily life activity to reduce rate of falls in older people (the LiFE study): randomised parallel trial. BMJ. 2012 Aug;345 aug07 1:e4547.

13 Rose DJ. Physical activity instruction of older adults, 2e. Human Kinetics; 2018

14 Taraldsen K, Mikolaizak AS, Maier AB, Boulton E, Aminian K, van Ancum J, et al. Protocol for the preventit randomised controlled trial feasibility study of a lifestyle-integrated exercise intervention in young older adults. BMJ Open. 2019 Mar 20;9(3):e023526.

15 Craig P, Dieppe P, Macintyre S, Michie S, Nazareth I, Petticrew M; Medical Research Council Guidance. Developing and evaluating complex interventions: the new Medical Research Council guidance. BMJ. 2008 Sep; 337:a1655.

16 Stuck AE, Walthert JM, Nikolaus T, Büla CJ, Hohmann C, Beck JC. Risk factors for functional status decline in community-living elderly people: a systematic literature review. Soc Sci Med. 1999 Feb;48(4):445-69.

17 Bohannon RW, Bubela DJ, Magasi SR, Wang YC, Gershon RC. Sit-to-stand test: performance and determinants across the age-span. Isokinet Exerc Sci. 2010;18(4):235-40.

18 Mignardot JB, Deschamps T, Barrey E, Auvinet B, Berrut G, Cornu C, et al. Gait disturbances as specific predictive markers of the first fall onset in elderly people: a two-year prospective observational study. Front Aging Neurosci. 2014 Feb;6:22.

19 Shumway-Cook A, Woollacott MH. Motor control: Theory and practical applications. Lippincott Williams \& Wilkins; 2001.

20 Beurskens R, Bock O. Age-related deficits of dual-task walking: a review. Neural Plast. 2012;2012:131608.

21 Biswas A, Oh PI, Faulkner GE, Bajaj RR, Silver MA, Mitchell MS, et al. Sedentary time and its association with risk for disease incidence, mortality, and hospitalization in adults: a systematic review and meta-analysis. Ann Intern Med. 2015 Jan;162(2):123-32.
22 Clemson L, Munro J. Conceptual model of habit reforming to improve balance and prevent falls. In: Pachana N, editor. Encyclopedia of geropsychology. Singapore: Springer; 2017. pp. 587-96.

23 Boulton E, Hawley-Hague H, French DP, Mellone S, Zacchi A, Clemson L, et al. Implementing behaviour change theory and techniques to increase physical activity and prevent functional decline among adults aged 6170: the PreventIT project. Prog Cardiovasc Dis. 2019 Mar - Apr;62(2):147-56.

24 Gardner B, Sheals K, Wardle J, McGowan L. Putting habit into practice, and practice into habit: a process evaluation and exploration of the acceptability of a habit-based dietary behaviour change intervention. Int J Behav Nutr Phys Act. 2014 Oct;11(1):135.

25 Szanton SL, Walker RK, Roberts L, Thorpe RJ Jr, Wolff J, Agree E, et al. Older adults' favorite activities are resoundingly active: findings from the NHATS study. Geriatr Nurs. 2015 Mar-Apr;36(2):131-5.

26 Michie S, Richardson M, Johnston M, Abraham C, Francis J, Hardeman W, et al. The behavior change technique taxonomy (v1) of 93 hierarchically clustered techniques: building an international consensus for the reporting of behavior change interventions. Ann Behav Med. 2013 Aug;46(1):81-95.

27 Clemson L, Munro J, Fiatarone Singh MA. Lifestyle-integrated functional exercise program to prevent falls: Trainer's manual. Sydney University Press; 2014.

28 Wulf G. Attention and motor skill learning. Human Kinetics; 2007.

29 Shigematsu R, Okura T, Nakagaichi M, Tanaka K, Sakai T, Kitazumi S, et al. Square-stepping exercise and fall risk factors in older adults: a single-blind, randomized controlled trial. J Gerontol A Biol Sci Med Sci. 2008 Jan; 63(1):76-82.

30 Sun F, Norman IJ, While AE. Physical activity in older people: a systematic review. BMC Public Health. 2013 May;13(1):449.

31 Clemson L, Munro J, Fiatarone Singh MA. Lifestyle-integrated functional exercise program to prevent falls: Participants manual. Sydney University Press; 2014.

32 Nasreddine ZS, Phillips NA, Bédirian V, Charbonneau S, Whitehead V, Collin I, et al. The Montreal Cognitive Assessment, MoCA: a brief screening tool for mild cognitive impairment. J Am Geriatr Soc. 2005 Apr;53(4): 695-9. 
33 Powell LE, Myers AM. The activities-specific balance confidence $(a b c)$ scale. J Gerontol A Biol Sci Med Sci. 1995 Jan;50A(1):M28-34.

34 Rikli RE, Jones CJ. Functional fitness normative scores for community-residing older adults, ages 60-94. J Aging Phys Act. 1999; $7(2): 162-81$.

35 Bischoff HA, Stähelin HB, Monsch AU, Iversen MD, Weyh A, von Dechend M, et al. Identifying a cut-off point for normal mobility: a comparison of the timed 'up and go' test in community-dwelling and institutionalised elderly women. Age Ageing. 2003 May;32(3): 315-20.

36 Weber M, Van Ancum J, Bergquist R, Taraldsen K, Gordt K, Mikolaizak AS, et al. Concurrent validity and reliability of the Community Balance and Mobility scale in young-older adults. BMC Geriatr. 2018 Jul;18(1):156.

37 Boulton E, Weber M, Hawley-Hague $H$, Bergquist R, Van Ancum J, Jonkman N, et al. Attitudes towards adapted Lifestyle-integrated Functional Exercise developed for 60- to 70-year-olds: perceptions of participants and trainers. Gerontology. Forthcoming 2019.

38 Zhang W, Schwenk M, Mellone S. Complexity of daily physical activity is more sensitive than conventional metrics to assess functional change in younger older adults. Sensors (Basel). 2018 Jun 25;18(7). pii: E2032.
39 Cohen J. Statistical power analysis for the behavioral sciences. Hillsdale, NJ: L. Lawrence Earlbaum Associates; 1988. p. 2.

40 Schoenfeld BJ, Ogborn D, Krieger JW. Effects of resistance training frequency on measures of muscle hypertrophy: A systematic review and meta-analysis. Sports Med. 2016 Nov; 46(11):1689-97.

41 Burton E, Lewin G, Clemson L, Boldy D. Effectiveness of a lifestyle exercise program for older people receiving a restorative home care service: a pragmatic randomized controlled trial. Clin Interv Aging. 2013;8:1591-601.

42 Liu CJ, Latham NK. Progressive resistance strength training for improving physical function in older adults. Cochrane Database Syst Rev. 2009 Jul;(3):CD002759.

43 Finch CF, Owen N. Injury prevention and the promotion of physical activity: what is the nexus? J Sci Med Sport. 2001 Mar;4(1):77-87.

44 Gardner B, Smith L, Lorencatto F, Hamer M, Biddle SJ. How to reduce sitting time? A review of behaviour change strategies used in sedentary behaviour reduction interventions among adults. Health Psychol Rev. 2016; 10(1):89-112.
45 Lesinski M, Hortobágyi T, Muehlbauer T, Gollhofer A, Granacher U. Effects of balance training on balance performance in healthy older adults: A systematic review and metaanalysis. Sports Med. 2015 Dec;45(12):172138.

46 Hillsdon M, Foster C, Thorogood M. Interventions for promoting physical activity. Cochrane Database Syst Rev. 2005 Jan; (1):CD003180.

47 Tudor-Locke C, Craig CL, Aoyagi Y, Bell RC, Croteau KA, De Bourdeaudhuij I, et al. How many steps/day are enough? For older adults and special populations. Int $J$ Behav Nutr Phys Act. 2011 Jul;8(1):80.

48 Copeland JL, Ashe MC, Biddle SJ, Brown WJ, Buman MP, Chastin S, et al. Sedentary time in older adults: a critical review of measurement, associations with health, and interventions. Br J Sports Med. 2017 Nov;51(21):1539.

49 Dunstan DW, Kingwell BA, Larsen R, Healy GN, Cerin E, Hamilton MT, et al. Breaking up prolonged sitting reduces postprandial glucose and insulin responses. Diabetes Care. 2012 May;35(5):976-83.

50 Jansen CP, Nerz C, Kramer F, Labudek S, Klenk J, Dams J, et al. Comparison of a groupdelivered and individually delivered lifestyleintegrated functional exercise (LiFE) program in older persons: a randomized noninferiority trial. BMC Geriatr. 2018 Nov; 18(1): 267. 